\title{
MODELING A-TYPE ATMOSPHERES: NLTE MODELS
}

\author{
Ivan HUBENY \\ Universities Space Research Association, NASA/GSFC, Code 681, \\ Greenbelt, MD 20771, USA \\ Thierry LANZ *) \\ NASA Goddard Space Flight Center, Code 681, \\ Greenbelt, MD 20771, USA
}

\begin{abstract}
A brief review on NLTE models of normal and chemically peculiar stars of spectral types A and late B is presented. We will concentrate on three basic question about NLTE modeling, namely i) Why (calculate NLTE models)? ii) How (to calculate NLTE models efficiently)? and iii) What (we learn from NLTE models already calculated)?
\end{abstract}

\section{NLTE MODELS: WHY?}

NLTE stands for non-local-thermodynamic-equilibrium (it is also sometimes referred to as non-LTE), and is aimed to describe a state of matter characterized by any kind of departure from LTE. In practice, however, departures from LTE are allowed only for number densities (populations) of a relatively small number of selected atomic energy levels. The remaining level populations and all the particle velocity distributions are then taken in LTE (Saha-Boltzmann and Maxwell distributions, respectively).

The title question can be answered from both methodological as well as practical viewpoints. Methodologically, the NLTE description is a fundamentally better description of the interaction of radiation and matter. LTE is an approximation which may, but does not need, to be actually valid. Therefore, if for nothing else, NLTE models can serve to justify the applicability of LTE models, which are easier to calculate and therefore much more widespread. One may argue that for normal and chemically peculiar stars of spectral type $A$ and $B$ there are other, more important physical phenomena actively playing a rôle, as for instance, diffusion, meridional circulation, magnetic fields, etc. This may certainly be true, but the radiation, and particularly the spectral line profiles, will always remain the only probes of the physical structure of distant objects. The experience gained in the last two decades has amply demonstrated that in order to fully explore the wealth of information contained in the stellar spectrum, NLTE is, at least in principle, important.

*) NRC-NASA Research Associate 
However, we would like to express a serious warning about a too simplistic approach to NLTE. Since NLTE is inherently a more complex description than LTE, it has to neglect many features that LTE can easily afford to include. It concerns, in particular, the effect of numerous metal lines, traditionally called line blanketing. As discussed in detail by Hubeny (1986), NLTE approach is very successful for explaining certain features in the far UV continuum of A-stars and for explaining the cores of strong lines, but NLTE model atmospheres which do not include the effect of metal line blanketing are not very useful for practical purposes. Therefore, despite the undisputable partial successes of NLTE models of $A$ and $B$ stars, virtually all researchers in the field use the Kurucz (1979; 1990; see also these proceedings) grid of LTE line blanketed models. The biggest challenge for the stellar atmospheric modellers is then to construct NLTE fully line blanketed models, which would finally answer a long-standing question of whether and to what extent are the LTE models satisfactory.

\section{Brief History}

NLTE studies relevant to A and late B stars until 1985 were reviewed by Hubeny (1986). Therefore, we will consider here only studies done later than 1986.

i) Model atmospheres: In recent years, there has been a significant development in the methodology of computing NLTE model atmospheres, with more and more involved treatment of line blanketing. One method, called the multi-frequency/multi-gray algorithm, was developed by Anderson (1985, 1987, 1989). The method uses an ingenious rearranging of individual frequency points into a small set of frequency blocks. The other method is an application of the accelerated lambda iteration (ALI) technique, worked out by Werner and collaborators (Werner 1986, 1987, 1989; Dreizler and Werner 1991).

However, these modern techniques were applied so far only to early B stars and hotter, or to the Sun (Anderson and Athay 1989), but no new results were obtained for A of late B stars. One of the aims of this paper is to stress that this situation is likely to be changed dramatically in the immediate future.

ii) Line formation: There were several studies in the category of a "restricted NLTE approach", namely assuming a model atmosphere as given and solving the detailed line formation for an individual atom/ion. The inherent assumption of this type of approach is that the chemical element under study is a "trace element", i.e. it reflects the structure of the atmosphere, but the atmospheric structure is assumed to be unaffected by the changes of level populations of this species.

- Gigas (1986) undertook a detailed study of NLTE line formation of Fe I and Fe II for Vega. Departures from LTE were considered for 79 levels of Fe I and 20 levels of Fe II. The results are conveniently expressed in terms of NLTE corrections, which give the ratio of abundances derived from a given spectral line with a given equivalent width under the assumption of LTE and NLTE, respectively. He has shown that the basic NLTE effect is a modification of the ionization balance of Fe I/Fe II - all the states of Fe I are systematically underpopulated with respect to LTE, while the Fe II levels are generally close to LTE. In the terminology of Hubeny (1986), this is a typical case C of NLTE effects (where the rate of photoionization is much larger than the rate of recombination - due to irradiation by a strong UV radiation).

- Freire-Ferrero et al. (1987) studied Mg II line formation for Vega and Sirius, using a relatively simple model of the $\mathrm{Mg}$ II atom, with 6 levels allowed 
to depart from LTE, but considering also the partial frequency redistribution for the $\mathrm{Mg}$ II resonance lines.

- Gigas (1988) performed a detailed study of $\mathrm{Mg} I+\mathrm{Mg}$ II, with 71 and 48 levels out of LTE, respectively, and of Ba II, using 42 NLTE levels. Again, he found an important systematic underpopulation of the $\mathrm{Mg} \mathrm{I}$ and $\mathrm{Ba}$ II states with respect to LTE.

- Cugier and Hardorp (1988) studied C I + C II, with 10 NLTE levels of C I and 6 levels of C II (together with two lowest levels of C III and the ground state of $\mathrm{C}$ IV). They confirmed the underpopulation of the $\mathrm{C} I$ levels found previously by Snijders (1977) and Hubeny (1981), and calculated detailed profiles of the C II resonance lines, using a simplified partial redistribution.

- Lemke $(1989,1990)$ used Gigas' $(1986,1988)$ model atoms for iron and barium and performed NLTE line formation calculations for a larger sample of main sequence $A$ stars. In addition, he has carried out a detailed study of NLTE line formation of He I (considering $36 \mathrm{NLTE}$ levels), and $\mathrm{Ca}$ I + Ca II (with 19 and 3 NLTE levels, respectively). NLTE effects turned out to be rather insignificant for helium, while potentially very important for calcium.

- Stürenburg and Holweger (1990) performed a detailed NLTE study of $\mathrm{C} I$ in the Sun and Vega, using a much more sophisticated model atom than used before - with 88 NLTE levels and 66 line transitions. They showed that the NLTE effects increase dramatically towards stronger lines. An important methodological conclusion of this paper is that truly detailed NLTE calculations, considering many levels and transitions out of LTE, are required for the accurate analysis of $\mathrm{C} I$ lines. They demonstrated that simplified NLTE approaches using only a few low-lying levels and few bound-bound transitions may give spurious results.

- Recently, Steenbock and Holweger (1992) carried out a study of NLTE line formation of $\mathrm{Al} \mathrm{I}$, using 26 NLTE levels.

- Finally, Takeda (these proceedings) undertook the most detailed NLTE calculations for C I, N I, and O I so far.

Summarizing, all these studies showed that NLTE effects are very important for reliable abundance determinations. In most cases, previous discrepancies in abundances derived from lines of different ionization stages disappear when the NLTE effects are taken into account. More generally, these studies were able to show for which spectral features and which atmospheric parameters is the LTE approach sufficient. On the other hand, all the above mentioned approaches use very simplified underlying model atmospheres. Some of them express explicitly an urgent need for better model atmospheres. Since in most cases the most important NLTE effect is the ionization shift, which in itself is critically sensitive to the background, ionizing radiation, it is clear that the model atmosphere used as a background for line formation studies should contain a reliable continuum radiation, particularly in the short-wavelength part of the Balmer continuum ( 900 to $2000 \AA$ ), where a large number of photoionization edges of many important species are located. However, this continuum radiation itself is subject to appreciable NLTE effects (Hubeny 1981, 1986) and, moreover, the effects of thousands of spectral lines (ubiquitous line blanketing!) should be taken into account. So, again, the most important goal is to construct NLTE line blanketed model atmospheres for A and late B stars. 


\section{NLTE MODELS: HOW? - TOWARDS NLTE LINE BLANKETING}

The two modern methods for computing model atmospheres mentioned above, Anderson's method and ALI-based methods, were already used with success to construct NLTE line blanketed model atmospheres. On the other hand, the method of complete linearization introduced by Auer and Mihalas (1969) remains still a viable method. Generally, while ALI methods require much less computational effort per iteration, the number of iterations needed is typically rather large, of the order of tens to even hundreds. In contrast, complete linearization, which requires many more operations per iteration, usually exhibits much faster convergence - cases where as few as 5 iterations produce a reasonably converged model are not exceptional. Moreover the convergence is global. Since the complete linearization method is essentially a multidimensional Newton-Raphson method, the computer time is dominated by matrix inversions; the order of corresponding matrices is given by the total number of physical quantities needed to describe the physical state of the atmosphere at a certain depth. The computer time scales roughly as $N I \times N D \times N N^{3}$, where $N I$ is the number of iterations needed to achieve a required accuracy, $N D$ is the number of discretized depths (typically 70 to 100), and $N N$ is the number of atmospheric state parameters (unknowns) per depth.

Generally, one may reduce the total computer time either by reducing the time per iteration, or by reducing the number of iterations, or both. The former objective may be achieved either by reducing the size of matrices, or by avoiding repeated matrix inversions. The first way, reducing the size of matrices, which also means reducing the number of unknowns, is straightforward but should be applied with great caution in order not to reduce also the physics of the problem!

All these possibilities were discussed recently by Hubeny and Lanz (1992), who have suggested two efficient acceleration algorithms: the so called Kantorovich variant of the Newton-Raphson method, and the $\mathrm{Ng}$ acceleration. The former consists in keeping the Jacobi matrix of the system fixed after a few (typically 2 or 3 ) "normal" iterations; the latter is a technique already used in linear radiative transfer problems, which accelerates the iteration process by using information not only from the last iteration, but from the several previous iterations. Both methods, and particularly their synergistic combination, offer a significant improvement of the standard complete linearization.

We have appropriately modified the computer program TLUSTY (Hubeny 1988) to include the recent acceleration techniques. The program TLUSTY has already contained several ways of reducing the number of unknowns. The most important one is dividing of all atomic transitions into two groups, "explicit" transitions and "fixed-option" transitions. The radiation field in both groups of these transitions is treated essentially exactly, but is not linearized for the fixed transitions. Specifically, radiative rates (and also net cooling rates, and contributions to the total radiation pressure) for the fixed transitions are recalculated after each completed iteration of complete linearization, and are held fixed during linearization. It is clear that one cannot easily treat all transitions in the fixed mode, because in this case one would recover an essentially lambda-iteration type behavior, which is well known to converge extremely slowly, and even worse, tends to stabilize before the true convergence is reached (see, e.g. Mihalas 1978). Hubeny and Lanz (1992) showed, however, that with the new acceleration techniques the idea of fixed rates becomes even 
more attractive, because the Kantorovich acceleration allows one to perform very many iterations without an appreciable increase of computer time, and the $\mathrm{Ng}$ acceleration prevents the stabilization problems.

Nevertheless, NLTE line blanketed model atmospheres require that the great majority of transitions be treated with fixed rates. Our experience showed that even the above accelerations do not provide a fast and robust enough technique. Therefore, we have further improved the treatment of fixed transitions by applying the accelerated lambda iteration technique. In the new version, even the "fixed rates" are linearized; the only quantity kept fixed is the approximate lambda operator $\Lambda^{*}$. The new method is therefore a hybrid method between the complete linearization and ALI methods. Test cases show that one can now converge even a model atmosphere where all lines, including hydrogen Lyman lines, are taken in the "fixed" mode. All the models presented in this paper were calculated with this new technique. However, space available here does not allow us to go to any detail; a description of the method will be published elsewhere.

\section{NLTE Opacity Distribution Functions}

All these techniques are still insufficient to treat the $10^{4}$ to $10^{6}\left(10^{7}, 10^{8}, \ldots-\right.$ see paper by Kurucz in these proceedings) spectral lines, needed to deal with true line blanketing, individually. Therefore, one has to resort to some kind of statistical approach. Actually, even LTE models face the same problem. However, the situation is much easier there, because individual spectral lines do not need to keep their identity: an individual line is relevant only to the extent of how much opacity it provides. This opacity essentially depends only on temperature and electron density (and on the corresponding elemental abundance). Details of line broadening brings some complications, but in any case the opacity in the case of LTE is local. The problem of accounting for millions of spectral lines may easily be solved by introducing the opacity distribution function (ODF), which represents a resampled total opacity in a given frequency interval. All the lines of all different chemical species in a given spectral region may therefore be put into one ODF.

In contrast, in NLTE, the individual spectral lines are allowed to contribute not only to total opacity, but also, via their radiative rates, to the global statistical equilibrium (i.e. determination of atomic level populations). Moreover, numerous metallic lines influence radiative rates (mainly rates in bound-free transitions) of all other chemical species. And, finally, since the level populations no longer depend only on local conditions, but through radiation rates on physical conditions in deeper layers, the problem is inherently non-local and highly non-linear. In other words, it seems hopeless.

However, it is possible to adopt a statistical approach, while still keeping, to a certain extent, the identity of individual lines. The idea is not to deal with all the energy levels of complicated metal species separately. Instead, several states, which i) have the same parity, and ii) have very close energies, are grouped together to form a "superlevel". The basic physical assumption of this approach is that all the individual levels within a superlevel share the common NLTE departure coefficient. The optimum choice of partitioning of the individual levels into a reasonable set of superlevels is a matter of some experimentation, but in any case one ends with of the order of tens of superlevels. For example, our model represents Fe II by 31 superlevels (see Fig. 1). The 


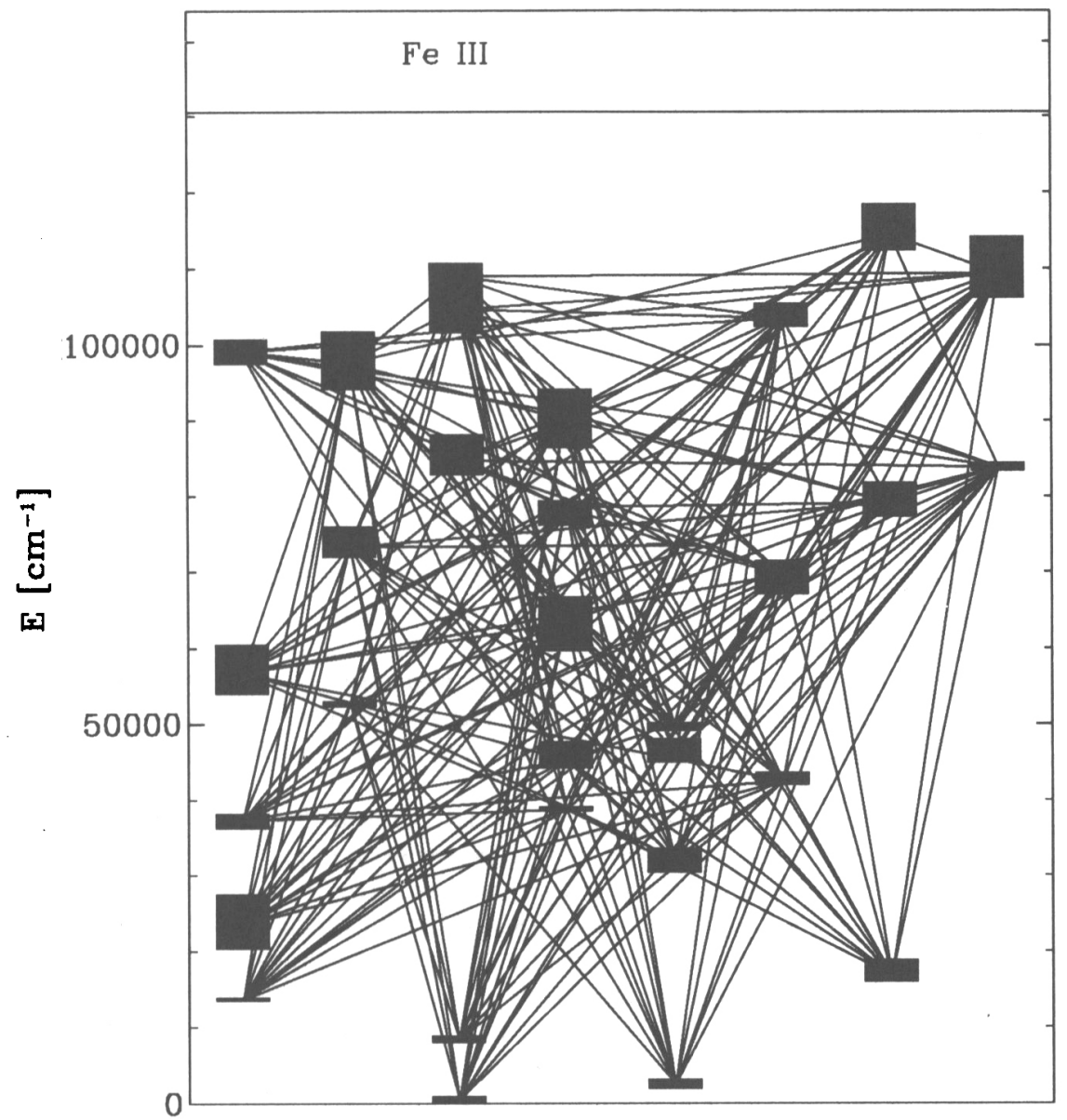

Fig. 1. Grouped energy levels (superlevels) and the corresponding superlines of our Fe II model atom. The $y$-axis is the level energy, while the position on the $x$-axis does not have any well-defined physical meaning except that the 1st, 3rd, etc., columns represent the even-parity states, and the rest the the odd-parity states. The boxes representing superlevels reflect the actual spread of energies of the individual components of the superlevel.

transitions between superlevels, called "superlines", may obviously be rather broad and their corresponding absorption cross-section is a highly nonmonotonic function of frequency. But since we do not need to keep the identity of individual lines, but only the individual superlines, one may construct for each superline a corresponding opacity distribution function. This is now a monotonic function of frequency, and therefore representable by a relatively very small number of frequency points. We have therefore call this approach NLTE opacity distribution function formalism. This idea is not new; it was used by Anderson (1989), and later by Dreizler and Werner (1992). Our approach differs mainly 


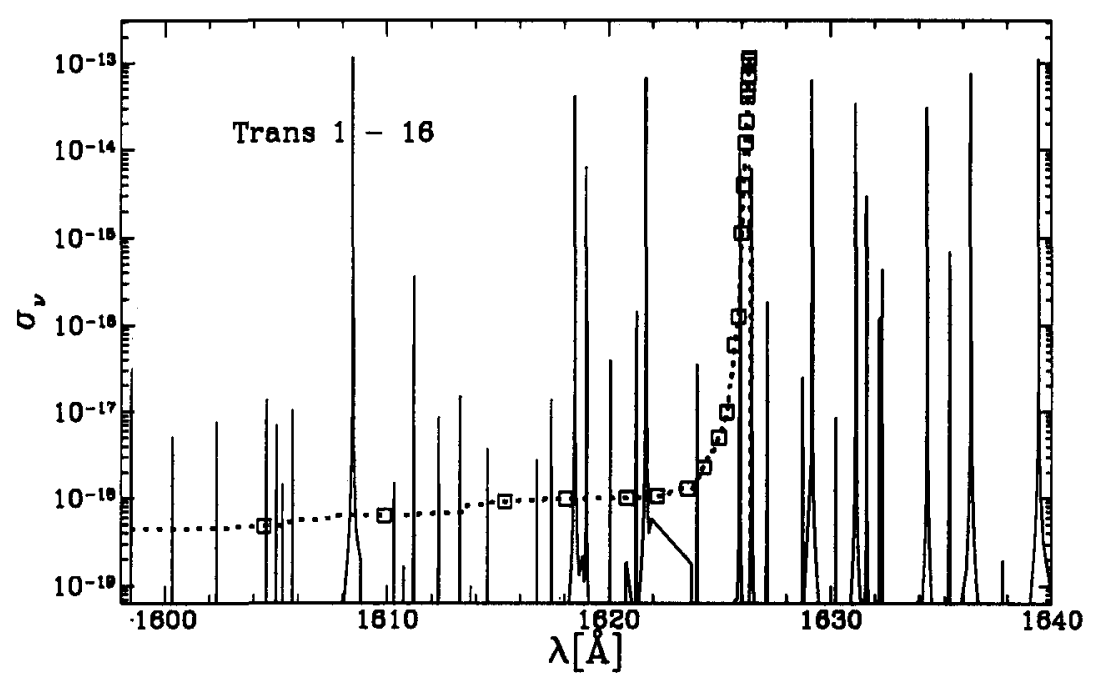

Fig. 2. The cross-section for a typical superline, and its representation by the opacity distribution function. The corresponding frequency points used in the model atmosphere construction are displayed as small squares.

in a more systematic way of choosing the partitioning of levels into superlevels, in a treatment of opacity sampling within a superline, and, in particular, in the treatment of an overlap of different superlines. Briefly, many superlines are actually represented by more that one ODF, taking into account both the case of their strong overlap, as well as the fact that a superlevel may have components on both sides of a strong continuum discontinuity, as for instance $\mathrm{C} I$ edge at $1100 \AA$. A detailed description of the method will be presented elsewhere.

Our basic goal was to construct a NLTE line blanketed model for an early A-type star. Since for these stars, by far the most important source of metal line opacity are lines of $\mathrm{Fe}$ II, we have limited ourselves in this paper only to Fe II. The model atom, showing the 31 superlevels, with their actual energy widths, and the corresponding 221 superlines, are shown in Fig. 1. An example of a detailed structure of a superline, together with the corresponding ODF and its discrete representation, is displayed in Fig. 2.

\section{NLTE MODELS: WHAT? - SOME RESULTS}

All the models presented below are calculated for $T_{\text {eff }}=10000 \mathrm{~K}, \log g=4$. These parameters are traditionally used to describe an archetype early A-type atmosphere. Also, this model is very interesting from the purely theoretical point of view, since the construction of a NLTE model, even for the simplest purehydrogen composition, presents a non-trivial problem, as the history of previous attempts attests (Frandsen 1974; Borsenberger and Gros 1978; Hubeny 1981).

We have calculated a number of models of various degree of sophistication, which will be discussed in detail in several forthcoming papers. A preliminary 
discussion of pure-hydrogen models was presented by Lanz et al. (1992). Here, we will only briefly mention few interesting examples.

\section{Hydrogen-carbon model atmospheres}

First, we have calculated almost "classical" NLTE model with hydrogen and carbon treated explicitly. For hydrogen, the first 8 levels were treated explicitly (in NLTE); the remaining levels were treated using the NLTE occupation probability formalism, which represents a generalization of the occupation probability formalism developed by Hummer and Mihalas (1988). It means, in practice, that no arbitrary separation of "existing" and "dissolved" levels is made; instead, all levels have a well-defined probability of being dissolved. A complex opacity near the Lyman and Balmer discontinuities is treated using the NLTE opacity distribution functions mentioned above. All the hydrogen lines, including Lyman lines, are taken into account. For C I, only 3 lowest levels were considered for these simple demonstration purposes.

Figure 3 displays the temperature structure for four models with increasing complexity: LTE model; NLTE/C model (continua only model - all lines are assumed to be in detailed radiative balance); NLTE/L model where all but Lyman lines were taken into account, and, finally, the model with all hydrogen lines - NLTE/LL. The behavior of first three models is well understood (Mihalas 1978; Hubeny 1981): temperature rise at $\log m<-3$ in the NLTE/L model is an indirect effect on Balmer lines on Balmer continuum. The model NLTE/LL was never calculated before due to previous serious numerical problems which were overcome only recently thanks to new techniques mentioned earlier. A dramatic temperature drop at $\log m \simeq-6$ is a result of the Lyman lines (mostly Lyman $\alpha$ ) cooling.

Figure 4 shows the corresponding theoretical UV continuum. The difference between LTE and NLTE models is primarily due to significant underpopulation of C I ground-configuration levels, discussed already by Snijders (1977) and Hubeny (1981). However, in contrast to what was believed earlier, differences between the UV flux for models NLTE/C and NLTE/L is appreciable. Figures 5 and 6 display the most important NLTE effects - line profiles for $\mathrm{H} \alpha$ and Lyman $\alpha$ in the core region. As expected, $\mathrm{H} \alpha$ is described well by the model NLTE/L, while in order to obtain a reliable Lyman $\alpha$ profile, the most sophisticated NLTE/LL model is mandatory.

\section{Influence of lines on the surface temperature}

One of the most important domains where NLTE description is crucial are the uppermost layers of an atmosphere. To demonstrate this phenomenon, we have constructed a series of models by adding more and more strong resonance lines into the model. The resulting temperature structure is depicted in Fig. 7. Model $A$ is the NLTE/C model, and B the NLTE/L model discussed above. Model $\mathrm{C}$ includes effect of C I and, in particular, C II resonance lines. The carbon line cooling increases gradually toward the surface. Next, model D includes, in addition to all the previous opacities, also the $\mathrm{Mg} \mathrm{II}$ resonance lines. Notice a significant cooling at $\log m \simeq-4$, which removes almost half of the heating produced by the Balmer lines. Next, model $E$ adds hydrogen Lyman lines, which again cause a dramatic cooling for $\log m \leq-6$. Finally, model F includes, in addition to all previous lines, also resonance lines of Fe II (13 superlines from superlevel 1, corresponding to 1021 individual lines). As expected, the Fe II 


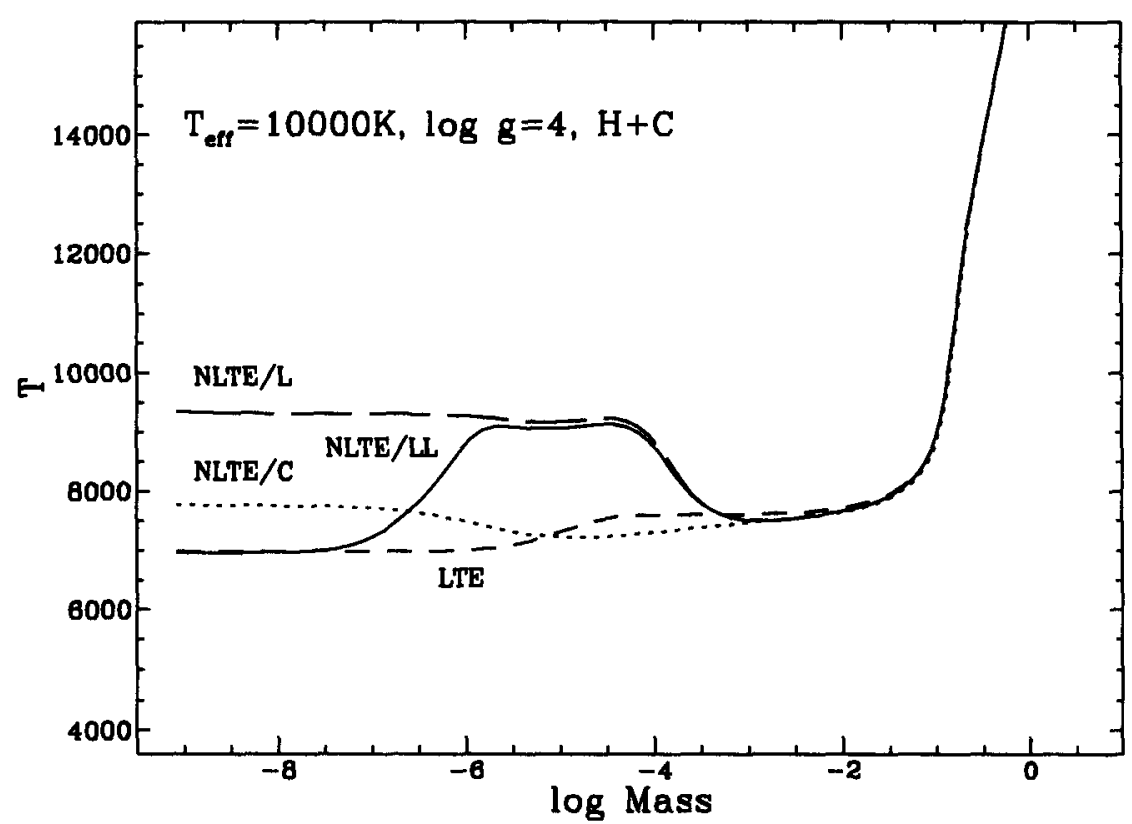

Fig. 3. Temperature structure for various hydrogen-carbon model atmospheres:

LTE model (short-dashed line); NLTE/C model (dotted line); NLTE/L (long-dashed line); and NLTE/LL model (full line). See the text for explanation of the model labels.

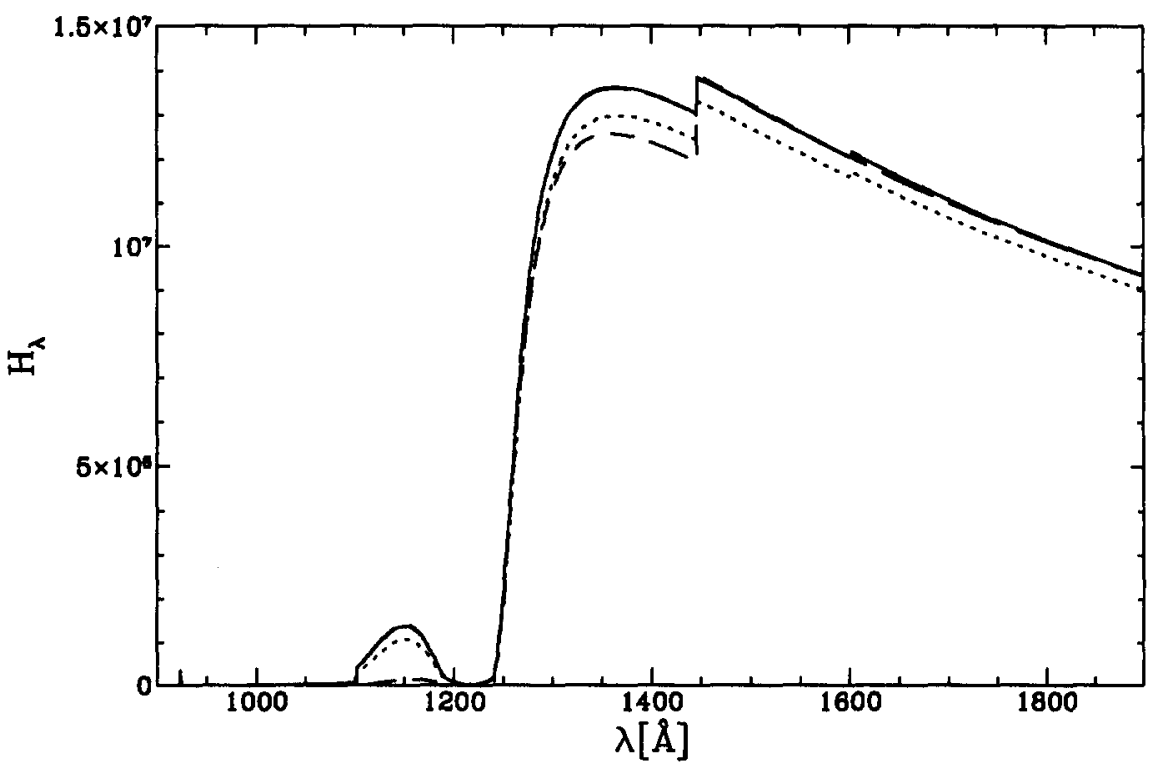

Fig. 4. UV continuum flux for the models displayed in Fig. 3. 


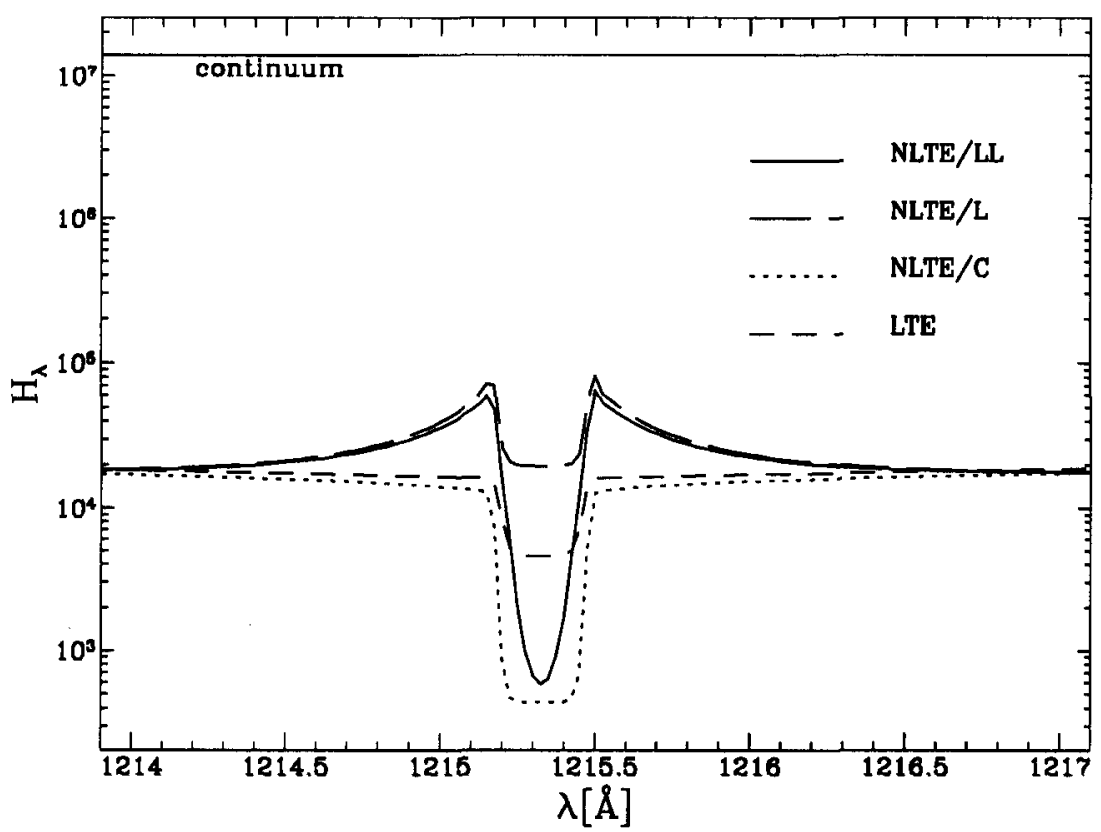

Fig. 5. Lyman $\alpha$ profile for the models displayed in Fig. 3.

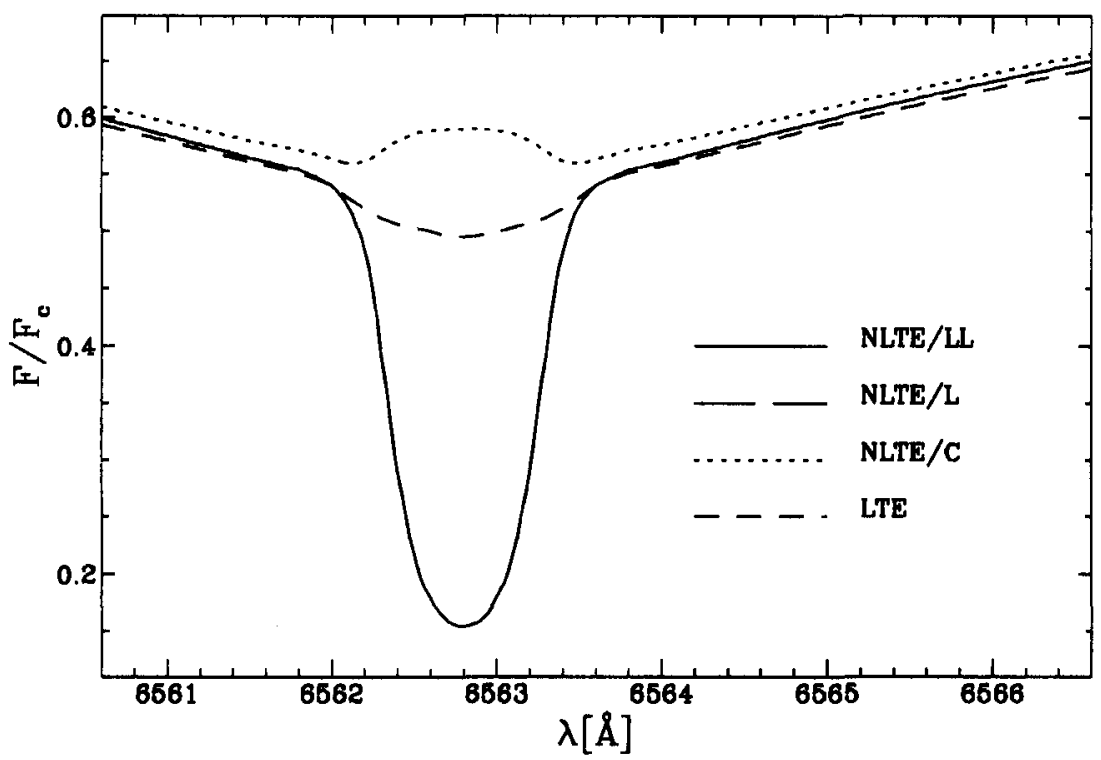

Fig. 6. $\mathrm{H} \alpha$ profile for the models displayed in Fig. 3. Notice that the profiles for the NLTE/L and NLTE/LL models coincide. 


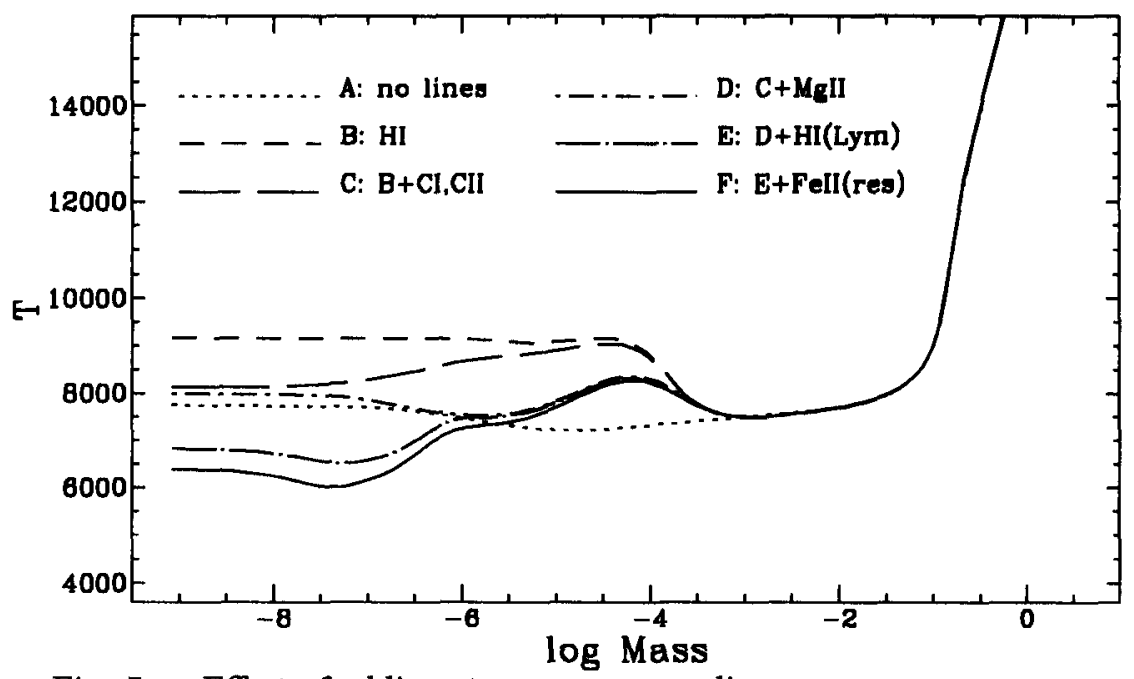

Fig. 7. Effect of adding strong resonance lines.

resonance lines produce a systematic cooling, but as we discuss below, the effect of resonance lines of Fe II is only a small part of the total effect of Fe II opacity.

Generally, Fig. 7 clearly shows that the predicted surface structure of an atmosphere is dramatically sensitive on the quality of the model, namely on the choice of lines treated in detail. Since for all the lines included here we found strong NLTE effects, NLTE description is clearly indispensable when dealing with these layers. We stress that the question of predicted radiative-equilibrium surface structure of an atmosphere is extremely important from the point of view of understanding energetics of possible chromospheres of early A stars. This point is discussed more by Lanz and Hubeny (these proceedings).

\section{Fe II line blanketed models}

Finally, we present some models with Fe II lines. An instructive example of LTE models is displayed in Fig. 8. Here, we constructed models composed of hydrogen, carbon ( 3 levels), and iron, increasing the number of Fe II lines considered in the model construction. Model A has no Fe II lines (only Fe II continua, which have, in fact, a negligible effect); model B contains $1927 \mathrm{Fe}$ II lines (all the superlines from the first two superlevels); model $\mathrm{C}$ has 12668 lines (all superlines from first 8 superlevels); and finally model D considers 45815 lines, corresponding to all our 221 superlines. We would like to stress here that we have used the Kurucz (1990) linelist and selected only lines originating between observed energy levels. There is in principle no problem to consider much more individual (predicted) lines here - of the order of $10^{6}$ to $10^{7}$, because all the remaining predicted levels would form only a few new superlevels, with a consequent modest increase of the number of superlines. Therefore, there would be no dramatic increase of computer time for computing a model (time would increase only in the preliminary step - an actual evaluation of the individual ODF's).

Figure 8 nicely demonstrates well-known classical features: both the backwarming (a temperature rise around $\log m \simeq-1$ ), and the surface cooling 


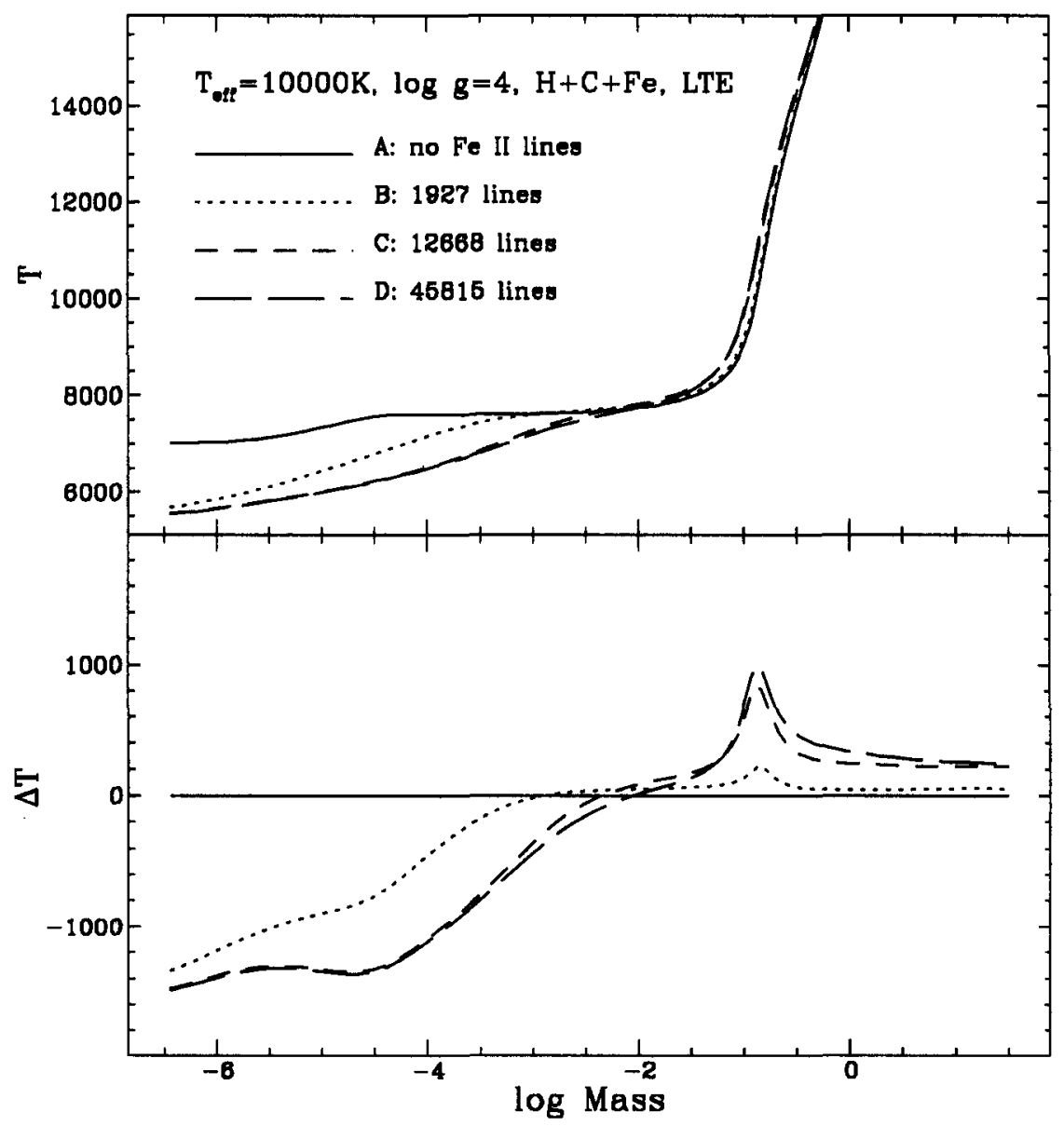

Fig. 8. Effect of increasing the number of $\mathrm{Fe}$ II lines: $\mathrm{A}-$ no $\mathrm{Fe}$ II lines; B - 1927 lines; C - 12668 lines; and D - 45815 lines. The upper panel shows the absolute temperature, the lower panel displays is the temperature difference from the model $A$.

increase with increasing number of lines. As expected, most cooling at the surface is provided by the resonance lines, while higher lines are the most effective coolants around $\log m \simeq-4$. However, the most interesting feature is that the difference between models $\mathrm{C}$ and $\mathrm{D}$ is already very small. In other words, the first some 13000 lines give the most of the total effect! In our opinion, this gives a strong support to our view that the crucial problem in the model atmosphere construction is not how many millions of lines one includes, but rather how well one considers the most important few tens of thousands of lines. However, the present figure represents only a very preliminary study. We plan to perform many more test calculations along these lines and to actually find the number 


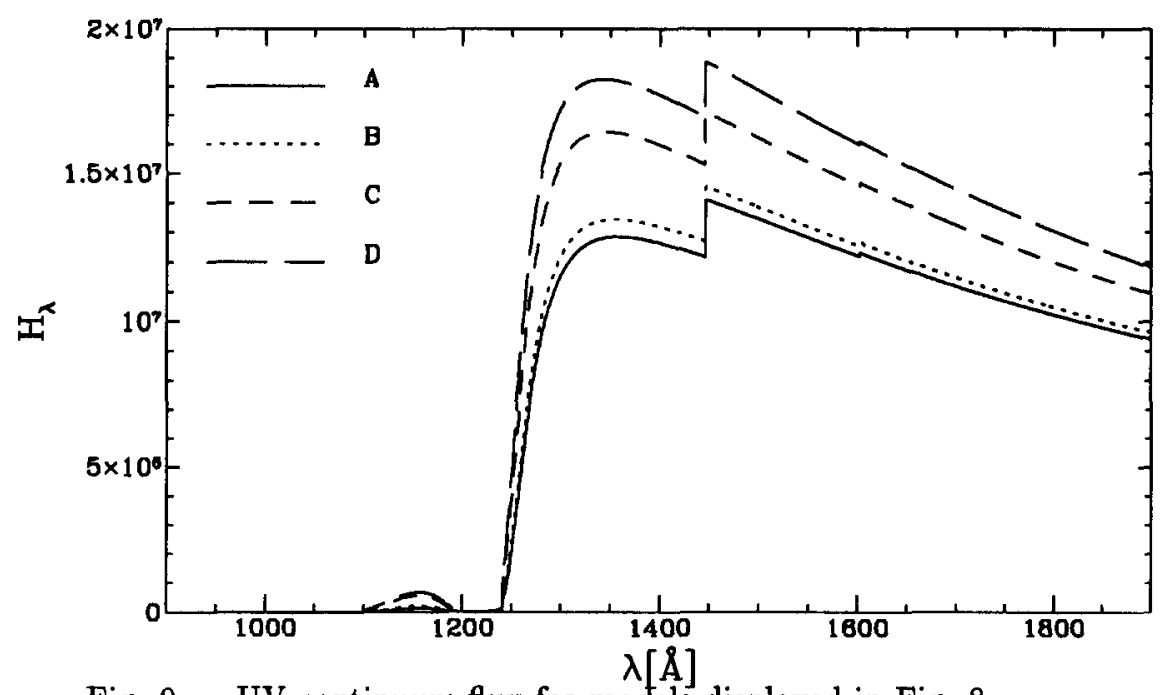

Fig. 9. UV continuum flux for models displayed in Fig. 8.

of Fe II lines which are to be included depending on the required accuracy of the resulting temperature structure.

Figure 9 displays the corresponding UV flux for the above models. It clearly shows a large sensitivity of the continuum flux on the degree of line blanketing used in the model construction. Actually, the UV continuum flux is more sensitive to the number of Fe II lines treated than to the difference between LTE and NLTE (compare to Fig. 4). This does not mean that NLTE is unimportant, but it again demonstrates what we stated at the beginning: unblanketed NLTE models neglect so much of the important physics (line blanketing) that they are actually worse than the LTE models.

Finally, as a conclusion, we compare four models: our fully blanketed $\mathbf{H}+$ $\mathrm{C}+\mathrm{Fe}$ II model (model D of Fig. 9); Kurucz (1990) model for the same effective temperature and gravity; a "classical" NLTE model (H + C NLTE/L model, without Lyman lines); and our current most involved NLTE line blanketed model including $\mathrm{H}, \mathrm{C} \mathrm{I}, \mathrm{C}$ II, Mg II, and Fe II (resonance lines only). Since the methodology was developed just few weeks before the meeting, and our computer program is still under active development and extensive testing, we were unable to calculate a full NLTE model with all Fe II lines. This will be presented in a future paper.

Nevertheless, we may draw several important conclusions even from these preliminary results: i) The agreement between our LTE model and the Kurucz model is very good. Actually, we are not aware of any other calculation of a line blanketed model atmospheres done with an absolutely independent computer program, since the overwhelming majority of workers use either Kurucz program ATLAS (Kurucz 1970) or its minor modifications. The remaining difference between our model and the Kurucz one is due to the fact that we included only Fe II as a source of line blanketing, and, secondly, that we adopted zero microturbulence in contrast to the value of $2 \mathrm{~km} / \mathrm{s}$ adopted by Kurucz. Since we did not aim to reproduce exactly the Kurucz model, the agreement may be viewed as excellent. ii) The difference between the classical NLTE model, and 


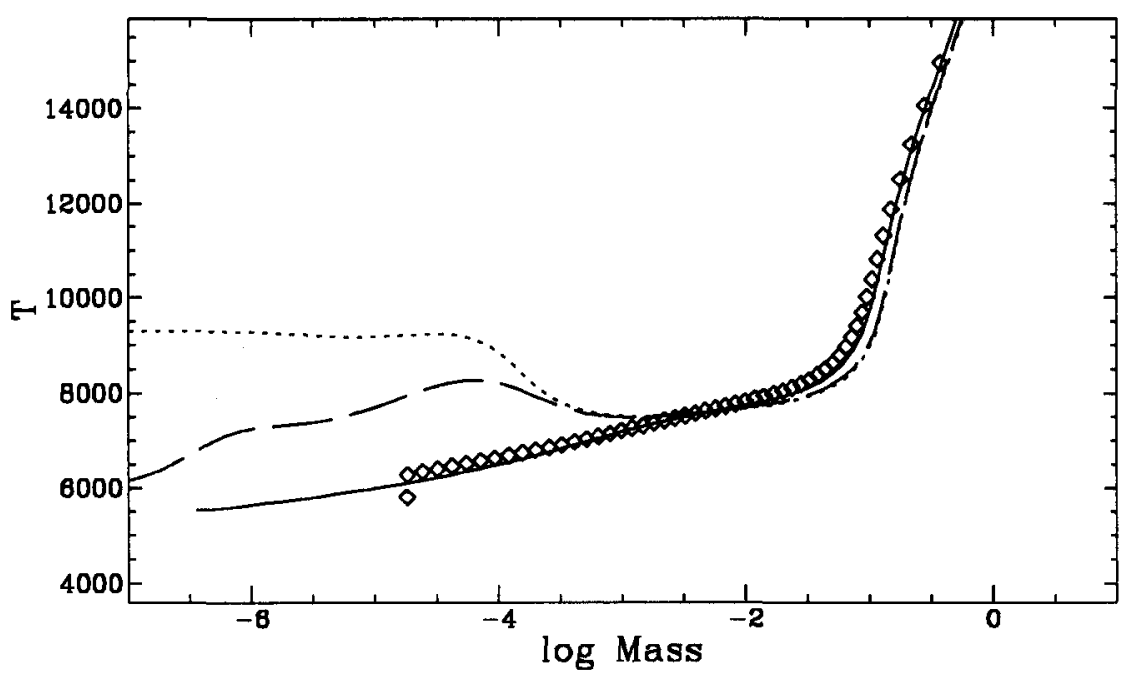

Fig. 10. Comparison of our LTE line blanketed model (full line) and the Kurucz model (diamonds); and two NLTE models: a "classical" NLTE/L hydrogen-carbon model (dotted line), and a NLTE line blanketed model (dashed line).

the model with some Fe II line blanketing is appreciable. iii) Finally, and most importantly, the differences between LTE blanketed and NLTE blanketed model are still very important. The difference in temperature in the continuum forming layers (around $\log m=-1$ ) are most likely due to still insufficient number of lines in our NLTE model (compare to Fig. 8 which shows that considering next 13000 Fe II lines produces an additional backwarming very close to the missing one). However, temperature difference in the outermost layers will most likely remain even when more Fe II lines will be added. Again, results shown in Fig. 8 serve as a guide of what one may expect.

\section{CONCLUSIONS}

The recent dramatic advances in the fast numerical methods for solving the radiative transfer problem have spurred an impressive progress in stellar atmospheric modeling. We are now becoming able to calculate truly NLTE line blanketed model atmospheres, with an unprecedented degree of realism and accuracy. After the epoch when researchers were aware of the potential importance of the NLTE models for A-type stars but were unable to produce model atmospheres which could compete with published extensive grids of LTE line blanketed models, we now entered the epoch when a construction of a grid of NLTE line blanketed atmospheres is no longer a dream, but is becoming a reality.

Acknowledgements: We thank Sally Heap for a careful reading of the manuscript. The work on this project was supported in part by the NASA grant No. 65 under program NRA 91-OSSA-12. 


\section{REFERENCES}

Anderson, L.S., 1985, ApJ 298, 848.

Anderson, L.S., 1987, In: Numerical Radiative Transfer, Kalkofen, W. (ed.), Cambridge University Press, Cambridge, p. 163.

Anderson, L.S., 1989, ApJ 339, 558.

Anderson, L.S., Athay, R.G., 1989, ApJ 346, 1010.

Auer, L.H., Mihalas, D., 1969, ApJ 158, 641.

Borsenberger, J., Gros, M., 1978, A\&AS 31, 291.

Cugier, H., Hardorp, J., 1988, A\&A 197, 163.

Dreizler, S., Werner, K., 1991, In: Stellar Atmospheres: Beyond Classical Models, NATO ASI Series C 341, Crivellari, L., Hubeny, I., Hummer, D.G. (eds.), Kluwer, Dordrecht, p. 155.

Dreizler, S., Werner, K., 1992, In: Atmospheres of Early-type stars, Lecture Notes in Phys., Heber, U., Jeffery, S. (eds.), Springer Verlag, p. 436.

Frandsen, S., 1974, A\&A 37, 139.

Freire Ferrero, R., Gouttebroze, P., Talavera, A., 1987, A\&A 173, 315.

Gigas, D., 1986, A\&A 165, 170.

Gigas, D., 1988, A\&A 192, 264.

Hubeny, I., 1981, A\&A 98, 96.

Hubeny, I., 1986, In: Upper Main Sequence Stars with Anomalous Abundances, IAU Coll. No. 90, Cowley, C.R., Dworetsky, M.M., Mégessier, C. (eds.), Dordrecht, Reidel, p. 57.

Hubeny, I., 1988, Comput. Phys. Commun. 52, 103.

Hubeny, I., Lanz, T., 1992, A\&A 262, 501.

Hummer, D.G., Mihalas, D., 1988, ApJ 331, 794.

Kurucz, R.L., 1970, SAO Spec. Rep. No. 309.

Kurucz, R.L., 1979, ApJS 40, 1.

Kurucz, R.L., 1990, in Trans. IAU 20B, 168.

Lanz, T., Hubeny, I., Altner, B., BAAS 23, 1382.

Lemke, M., 1989, A\&A 225, 125.

Lemke, M., 1990, A\&A 240, 331.

Mihalas, D., 1978, Stellar Atmospheres, Freeman, San Francisco.

Snijders, M.A.J., 1977, ApJ 214, L35.

Steenbock, W., Holweger, H., 1992, In: Atmospheres of Early-type stars,

Lecture Notes in Phys., Heber, U., Jeffery, S. (eds.), Springer Verlag, p. 57.

Stürenburg, S., Holweger, H., 1990, A\&A 237, 125.

Werner, K., 1986, A\&A 161, 177.

Werner, K., 1987, In: Numerical Radiative Transfer, Kalkofen, W. (ed.), Cambridge University Press, Cambridge, p. 67.

Werner, K., 1989, A\&A 226, 265. 